\title{
Assay of Antioxidant Capacity and Phenolic Compounds in some Romanian and Cypriot Wine
}

\author{
Camelia E. LUCHIAN ${ }^{1}$, Cintia L. COLIBABA ${ }^{1}$, Maria CODREANU², \\ Stefan TUDOSE-SANDU-VILLE ${ }^{1}$, Marius NICULAUA ${ }^{2}$, \\ Valeriu V. COTEA ${ }^{1 *}$

\begin{abstract}
${ }^{1}$ University of Agricultural Sciences and Veterinary Medicine Iași, 3 M. Sadoveanu Alley, 700490 Iași, Romania; kamelia_luchian@yahoo.com; cintia.colibaba@gmail.com; stefan_ville@yahoo.com;vvcotea@yahoo.com (*correspondingauthor)

${ }^{2}$ Romanian Academy of Sciences, Center of Oenological Researches, Iași Branch, 9 M. Sadoveanu Alley, 700490 Iași,

Romania;codreanu.maria@yahoo.com;niculaua@gmail.com
\end{abstract}

\begin{abstract}
Free radicals have an important role in food and in chemical material degradation, contributing to the occurrence of many human health problems, but the antioxidants can considerably delay or prevent the oxidation of easily oxidable substrates. The present research aimed to assess the antioxidant activity, expressed by the presence of polyphenols, flavonols, flavones, anthocyanidins and flavanols, in several Romanian and Cypriot wines. The wine phenolics content was analysed by highperformance liquid chromatograph (HPLC) Shimadzu equipped with two chromatographic columns. Higher concentrations were registered in all red wines. The antioxidant activity quantification was carried out by the DPPH method, a simple and cheap approach based on the absorbance decrease determination of the DPPH radical (2,2-diphenyl- 1- picrylhydrazyl) in the presence of antioxidants. The highest antioxidant activity for white wines was determined at 'Spouriko' for Cypriot wine from 2013 (EC $50=1 / 38$ ) while for Romanian wines, the highest value was found in a 'Tămâioasă românească' (EC50 = 1/58) and for red wines at 'Maratheftiko' wine from $2012($ EC50 = 1/680) and in 'Fetească Neagră' wine from 2014 (EC50 =1/590). This study provides relevant information to consumers and industry alike regarding the beneficial role wine plays for human health. It also can act as a baseline for choosing a certain product, according to its sanogenic potential.
\end{abstract}

Keywords: antioxidant activity, DPPH method, phenolic compounds, Cypriot wines, Romanian wines

\section{Introduction}

There is a worldwide agreement that anthocyanins, flavonols, catechins, and other flavonoids contribute to the wine colour and astringency, while it has also been demonstrated that they scavenge the excess radicals and mitigate oxidative stress. Therefore, they contribute to the anticarcinogenic, antiatherogenic, antiinflammatory, antimicrobial, and antioxidant activities of some fruits (Llaudy et al., 2004; Chang et al., 2012; Xu et al., 2012).

Among natural antioxidants, red wine has attracted particular interest due to a high content of biologically active compounds (Lopez-Velez et al., 2003; Tsai et al., 2004). The moderate consumption of wine, especially red wine, has also been associated with the reduction in mortality from cardiovascular diseases, an effect known as the "French Paradox" (Renaud and De Lorgeril, 1992). The polyphenolic compounds present in wines, which are known to have a high antioxidant capacity, are involved in several protective activities against some degenerative diseases such as cancers, cardiovascular diseases, chronic inflammation and thrombosis (Bell et al., 2000; Scalbert et al., 2005; Majo et al., 2008; Sun et al., 2009; Xia et al., 2010). Hence, the beneficial properties of wines have been mainly interpreted based on the antioxidant properties on the flavonoid fraction, which are related to free radical scavenging (Cao and Prior, 2000). Non-flavonoid compounds are presented mainly in the pulp of the grapes, and the flavonoid compounds are found in the skins, seeds, and stems of grapes (Cotea et al., 1985). The phenolic composition of wines is conditioned by the grape variety and by other factors that influence the berry development, such as soil, geographical location, weather conditions (Rotaru et al., 2013) or management practices (Bunea et al., 2012). Once grapes are crushed, condensation reactions, which involve especially anthocyanins, catechins and procyanidins, take place, resulting in the formation of new 
pigments, which are responsible for wine colour changes. Winemaking techniques also play an important role in the extraction of polyphenols from grapes and in their further stability in wines; the time of maceration and fermentation in contact with the grape skins and seeds, pressing, maturation in oak, fining, and bottle aging influence the phenolic composition of wines (Cotea et al., 2010).

The content of phenolic substances and total antioxidant activity of the sets of samples are high correlated as many studies described (Arnous et al., 2002; Katalinić et al., 2004; Hua et al., 2009; Mitić et al., 2010). Several in vitro methods have been developed to measure antioxidant capacities of food, beverages and biological samples. The most commonly used antioxidant capacity assays were 1,1diphenyl-2-picrylhydrazyl radical (DPPH•) assay (Bondet et al., 1997); 2,2-azino-di-(3-ethylbenzothialozinesulphonic acid) (ABTS) assay (Re et al., 1999); ferric ion reducing antioxidant power (FRAP) assay (Benzie et al., 1996; Pulido et al., 2000); cupric ion reducing capability (CUPRAC) assay (Apak et al., 2004) and oxygen radical absorbance capacity (ORAC) assay (Cao et al., 1996; Naguib, 2000).

The DPPH method is a rapid and simple method for estimating the antiradical activity of foods using the stable free radical 1,1- diphenyl-2-pycrylhydrazyl (DPPH•) by the addition of scavenging compounds. This is one of a few stable and commercially available organic nitrogen radicals and shows a characteristic UV-Vis spectrum with a maximum absorbance close to $515 \mathrm{~nm}$ (Saint-Cricq de Gaulejac et al., 1999; Da Porto et al., 2000; Paixao et al., 2007).

All wine samples were analyzed for phenolic compounds content on a HPLC system Shimadzu Prominence 20 series (Castellari et al., 2002, Cotea et al., 2012).

The aim of this paper was to characterize the free radical scavenging activity using DPPH method (diphenyl-ppicrylhydrazyl radical) and HPLC analysis of phenolic compounds content of some commercial Romanian and Cypriot wines.

\section{Materials and Methods}

\section{Samples}

The present study has chosen 55 white, rosé and red wine samples (25 Cypriot and 30 Romanian) of different vintages and different areas of Cyprus and Romania. Cypriot wines for 2012 vintage of which 5 white and 10 reds, 15 for 2013 vintage, of which 9 whites and 5 reds. The distribution of analysed Romanian wines is as follows: a white wine vintage 2006, vintage 2011 with 3 whites, one red from 2012, vintage 2013 with 8 whites and 3 reds, vintage 2014 with 10 whites, 1 rosé and 2 reds. All were still wines. All wine samples are presented in Table 1.

The chosen grape varieties are well-known both for the Cypriot and the Romanian red, white and rosé winemaking, there for deemed important for this study.

\section{Reagents}

All used reagents have been purchased from SigmaAldrich Co (St. Louis, MO, USA). All other chemicals used were of analytical grade.
Determination of antioxidant activity/capacity of wines through diphenyl-p-picrylhydrazyl (DPPH) method

The original procedure (Sánchez-Moreno et al., 1995) was modified by using a platform for the antiradical depletion DPPH, made with a Visible or UV-Vis spectrophotometer, multi-plate reader M200 Pro (Tecan Group Ltd., Männedorf, Switzerland) with polymethyl methacrylate well plates. A series of dilution was needed to calculate the result. This dilutions were: Reference solution D0: $9 \mathrm{~mL}$ of the DPPH• methanolic solution $+100 \mu \mathrm{L}$ of $\mathrm{MeOH}$; dilution D1: $1 / 40$ dilution of wine $(4 \mathrm{~mL}$ of the $\mathrm{DPPH} \bullet+100 \mu \mathrm{L}$ wine); dilution D2: $1 / 80$ dilution of wine ( $4 \mathrm{~mL} \mathrm{DPPH} \bullet+50 \mu \mathrm{L}$ wine); D3: $1 / 160$ dilution of wine ( $4 \mathrm{~mL} \mathrm{DPPH} \bullet+25 \mu \mathrm{L}$ wine); D4: $1 / 320$ dilution of wine ( $4 \mathrm{~mL} \mathrm{DPPH} \bullet+12.5 \mu \mathrm{L}$ wine); D5:1/640 dilution of wine $(4 \mathrm{~mL} \mathrm{DPPH} \bullet+6.25 \mu \mathrm{L}$ wine). The antioxidant activity was evaluated based on free DPPH• radicals remaining in the medium after the reaction between the methanolic DPPH• solution and the tested samples took place. For each dilution from D0 to D5, the reduction in the absorbance was determined at $515 \mathrm{~nm}$ at $0 \mathrm{~min}$. and every $1 \mathrm{~min}$. for $14 \mathrm{~min}$., and every $10 \mathrm{~min}$. until the reaction reaches a plateau in about 1 hour. The antioxidant activity of the wine is thus defined by the dilution of wine required to decrease the initial concentration of $\mathrm{DPPH} \bullet$ by 50\%: Efficient Concentration = EC50. Under these conditions, the lower EC50 of a tested samples, the higher its antioxidant activity.

\section{HPLC phenolic compounds analysis}

For the phenolics content analysis (Castellari et al., 2002), the wine samples were processed on a Shimadzu HPLC system consisting of: quaternary pump Shimadzu Prominence LC-20AD with autoinjector SIL-20AC, diode array detector SPD $600 \mathrm{~nm}$, chromatographic system controller CBM connectivity via LAN. The column system was made of a pre Cartridges UHPLC C18 for $4.6 \mathrm{~mm} \mathrm{ID}$ coupled to columns manufactured by Phenomenex. The elution flow was $0.85 \mathrm{~mL} \mathrm{~min}^{-1}$ and the column compartment was set at $50{ }^{\circ} \mathrm{C}$. The amount of phenolic compounds in the extracts was calculated as $\mathrm{mg} / \mathrm{L}$ wine using external calibration curves, which were obtained for each phenolic standard.

\section{Statistical analysis}

As the data was not normally distributed, Spearman'sRho $\left(r_{s}\right)$ correlation coefficients were calculated in order to characterize the relationship between antioxidant capacities detected by DPPH assay and phenolics content quantified by HPLC method. Spearman's rank correlation coefficient is a measure of correlation, written in short as the Greek letter $r h o(\rho)$ or sometimes as $r_{s}$. It is a number that shows how closely two sets of data are linked. It only can be used for data that can be put in order, such as highest to lowest. The general formula for $r_{s}$ is:

$$
\rho=1-\frac{6 \Sigma \mathrm{d}^{2}}{n\left(n^{2}-1\right)}
$$

where:

$\mathrm{d}=$ difference in paired ranks and $n=$ number of cases. The following guide for the absolute value was used: $0.00-0.19$ "very weak correlation"; $0.20-0.39$ "weak correlation"; $0.40-0.59$ 
242

"moderate correlation"; $0.60-0.79$ "strong correlation"; 0.801.0 "very strong correlation", as mentioned in other research studies (Fenercioglu et al., 2010; Floegela et al., 2011; Harris et al., 2011).
All statistics were performed with Microsoft Excel 2000. Correlations were established using regression analysis at a 95, 99, and 99.9\% significance level. The P-value less than 0.05 were considered statistically significant.

Table 1. The Cypriot and the Romanian analysed wine samples

\begin{tabular}{|c|c|c|c|}
\hline Sample Code & Colour & Vintage & Grape variety \\
\hline \multicolumn{4}{|c|}{ Cypriot Wines } \\
\hline C-12-01 & White & 2012 & 'Promara' \\
\hline C-12-02 & White & 2012 & 'Chardonnay' \\
\hline C-12-03 & Red & 2012 & 'Merlot' \\
\hline C-12-04 & White & 2012 & 'Morokanella' \\
\hline C-12-05 & White & 2012 & 'Spouriko' \\
\hline C-12-06 & White & 2012 & 'Xynisteri' \\
\hline C-12-07 & Red & 2012 & 'Maratheftiko' \\
\hline C-12-08 & Red & 2012 & 'Giannoudi' \\
\hline C-12-09 & Red & 2012 & 'Maratheftiko' \\
\hline C-12-10 & Red & 2012 & 'Maratheftiko' \\
\hline C-13-01 & White & 2013 & 'Sauvignon Blanc' \\
\hline C-13-02 & White & 2013 & 'Promara' \\
\hline C-13-03 & White & 2013 & 'Promara' \\
\hline C-13-04 & White & 2013 & 'Morokanella' \\
\hline C-13-05 & White & 2013 & 'Chardonnay' \\
\hline C-13-06 & White & 2013 & 'Spouriko' \\
\hline C-13-07 & White & 2013 & 'Xynisteri' \\
\hline C-13-08 & Red & 2013 & 'Cabernet Franc' \\
\hline C-13-09 & Red & 2013 & 'Morokanella' \\
\hline C-13-10 & Red & 2013 & 'Ntopio Mauro' \\
\hline C-13-11 & Red & 2013 & 'Ofthalmo' \\
\hline C-13-12 & Red & 2013 & 'Maratheftiko' \\
\hline C-13-13 & White & 2013 & 'Xynisteri' \\
\hline C-13-14 & White & 2013 & 'Xynisteri' \\
\hline C-13-15 & Red & 2013 & 'Giannoudi' \\
\hline \multicolumn{4}{|c|}{ Romanian Wines } \\
\hline R-07-01 & White & 2014 & 'Grasă de Cotnari’ \\
\hline R- $-07-02$ & White & 2014 & 'Francușă’' \\
\hline $\mathrm{R}-07-03$ & White & 2014 & 'Fetească regală' \\
\hline R-07-04 & White & 2014 & 'Muscat Ottonel' \\
\hline R- $-07-05$ & White & 2014 & 'Tămâioasă românească’ \\
\hline $\mathrm{R}-07-06$ & White & 2014 & 'Aligoté' \\
\hline R- $-07-07$ & White & 2014 & 'Sauvignon blanc' \\
\hline R- $-07-08$ & White & 2014 & 'Traminer' \\
\hline R-07-09 & White & 2014 & 'Riesling italian' \\
\hline $\mathrm{R}-07-10$ & Rosé & 2014 & 'Busuioacă de Bohotin’ \\
\hline $\mathrm{R}-07-11$ & Red & 2014 & 'Pinot noir' \\
\hline $\mathrm{R}-07-12$ & Red & 2014 & 'Fetească neagră \\
\hline $\mathrm{R}-07-13$ & White & 2014 & 'Pinot gris' \\
\hline $\mathrm{R}-07-14$ & Red & 2014 & 'Băbească neagră' \\
\hline $\mathrm{R}-07-15$ & White & 2013 & 'Zghihară de Huși' \\
\hline $\mathrm{R}-07-16$ & Red & 2013 & 'Fetească neagră’ \\
\hline $\mathrm{R}-07-17$ & White & 2011 & 'Fetească albă’ \\
\hline $\mathrm{R}-07-18$ & White & 2013 & 'Fetească albă' \\
\hline R-02-01 & White & 2011 & ‘Tămâioasă românească’ \\
\hline R-02-02 & White & 2011 & 'Grasă de Cotnari' \\
\hline $\mathrm{R}-02-03$ & White & 2013 & 'Fetească albă’ \\
\hline R-02-04 & White & 2013 & 'Francușă’ \\
\hline $\mathrm{R}-02-05$ & White & 2006 & 'Tămâioasă românească’ \\
\hline R-02-06 & White & 2013 & 'Aligoté' \\
\hline $\mathrm{R}-02-07$ & White & 2013 & 'Sauvignon blanc' Bio \\
\hline R-02-08 & White & 2013 & 'Fetească regală’' \\
\hline R-02-09 & White & 2013 & 'Busuioacă de Bohotin’ \\
\hline $\mathrm{R}-02-10$ & Red & 2013 & 'Băbească neagră' \\
\hline R-02-11 & Red & 2013 & 'Cabernet sauvignon' \\
\hline R-02-12 & Red & 2012 & ‘Cabernet sauvignon’ \\
\hline
\end{tabular}




\section{Results and Discussion}

The wines used in this study constituted a quite heterogeneous group, with different grape varieties, with diverse ages and ageing processes, therefore they showed important differences. Antioxidant activity results expressed as EC50 of different types of wines (red wine, white wine and rose wine) determined by the DPPH method are shown in Table 2. The obtained results correlate well with other literature finds: The method applied to samples of red wines shows that the efficient concentration factor EC50 varies approximately from $2.22^{*} 10^{-3}$ to $1.66^{*} 10^{-3}$. For white wines, the EC50 varies from $1.25^{*} 10^{-2}$ to $4^{*} 10^{-3}$. (BrandWilliams et al., 1995; Saint-Cricq de Gaulejac et al., 1999; Da Porto et al., 2000).

The obtained EC50 is inversely related to the antioxidant activity of a compound, as it expresses the amount of antioxidant needed to decrease the radical concentration by $50 \%$. The lower EC50, the higher the antioxidant activity of a compound is (Carmona-Jiménez et al., 2014). All wines scavenged DPPH• differently. Red wines were more active than whites. This can be attributed to their higher phenolic content.

Among the three wine colour groups, red Cypriot wines showed the highest antioxidant capacity, followed by rosés and whites (Fig. 1). Cypriot wines showed a higher antioxidant capacity than Romanian ones, on average.

Table 2. Effective concentration factor (EC50) for analysed wine samples

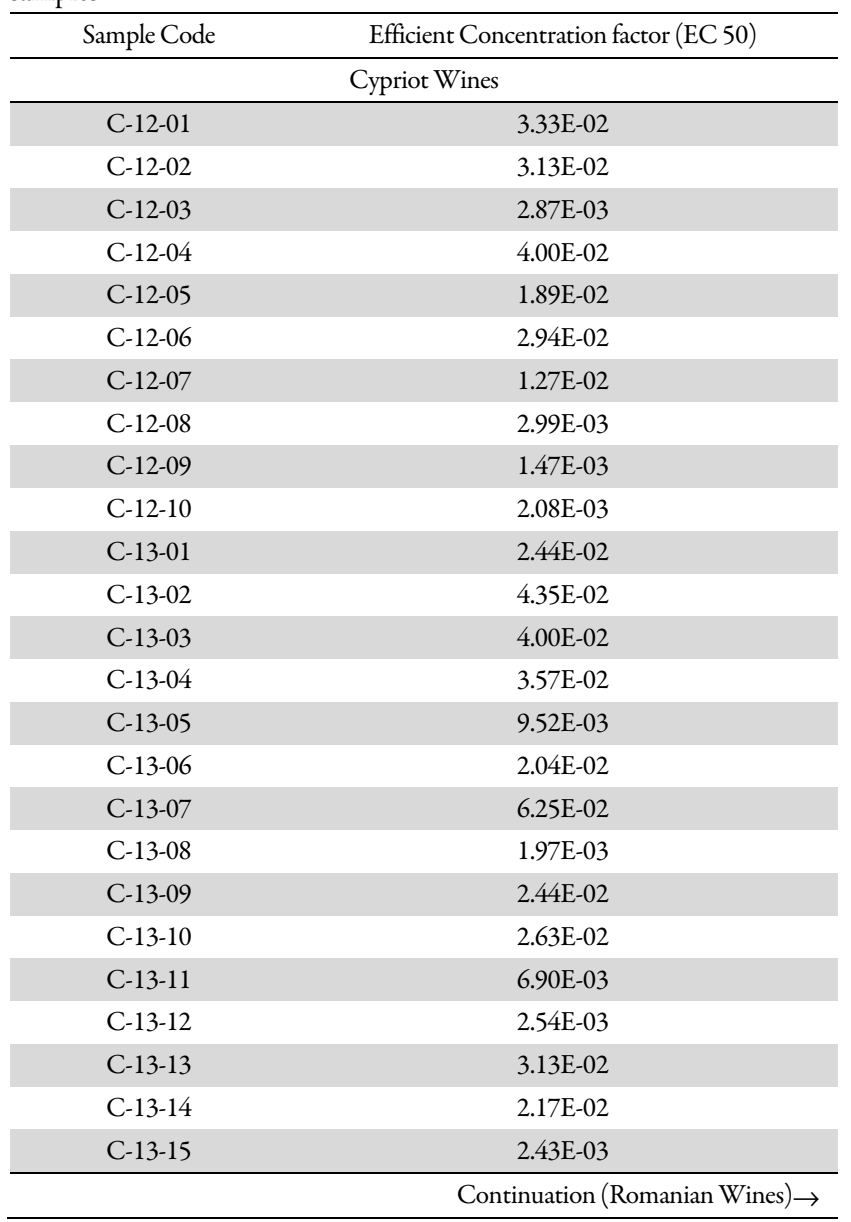

\begin{tabular}{|c|c|}
\hline \multicolumn{2}{|c|}{ Romanian Wines } \\
\hline R-07-01 & $6.25 \mathrm{E}-02$ \\
\hline R-07-02 & 6.67E-02 \\
\hline R-07-03 & $5.88 \mathrm{E}-02$ \\
\hline R-07-04 & 7.69E-02 \\
\hline $\mathrm{R}-07-05$ & $3.45 \mathrm{E}-02$ \\
\hline R-07-06 & 7.69E-02 \\
\hline R-07-07 & 8.33E-02 \\
\hline R-07-08 & 2.04E-02 \\
\hline R-07-09 & $7.69 \mathrm{E}-02$ \\
\hline R-07-10 & $2.08 \mathrm{E}-02$ \\
\hline R-07-11 & $1.64 \mathrm{E}-02$ \\
\hline R-07-12 & $1.81 \mathrm{E}-03$ \\
\hline R-07-13 & $2.44 \mathrm{E}-02$ \\
\hline R-07-14 & $4.59 \mathrm{E}-03$ \\
\hline R-07-15 & $2.86 \mathrm{E}-02$ \\
\hline R-07-16 & $3.09 \mathrm{E}-03$ \\
\hline R-07-17 & $5.56 \mathrm{E}-02$ \\
\hline R-07-18 & $4.35 \mathrm{E}-02$ \\
\hline R-02-01 & $1.72 \mathrm{E}-02$ \\
\hline R-02-02 & $2.38 \mathrm{E}-02$ \\
\hline R-02-03 & $6.25 \mathrm{E}-02$ \\
\hline R-02-04 & $3.85 \mathrm{E}-02$ \\
\hline $\mathrm{R}-02-05$ & $2.78 \mathrm{E}-02$ \\
\hline R-02-06 & $3.23 \mathrm{E}-02$ \\
\hline R-02-07 & 4.76E-02 \\
\hline R-02-08 & $5.00 \mathrm{E}-02$ \\
\hline R-02-09 & $2.04 \mathrm{E}-02$ \\
\hline R-02-10 & 4.78E-03 \\
\hline R-02-11 & $2.62 \mathrm{E}-03$ \\
\hline R-02-12 & $5.24 \mathrm{E}-03$ \\
\hline
\end{tabular}

\section{ED50 for Romanian and Cypriot wines}

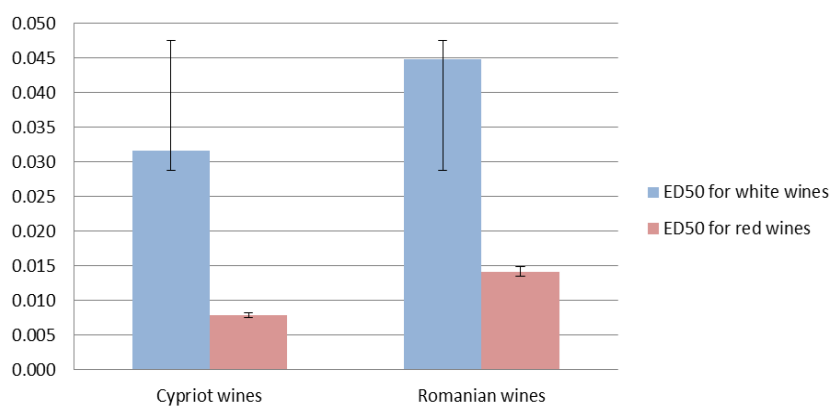

Fig. 1. Comparison of antioxidant capacities measured by DPPH assays, stratified by wine colour and wine nationality (mean and SD)

The HPLC analysis is the method used for the separation and quantification of a large variety of phenolic compounds from wine composition. The HPLC approach achieved 13 components including phenolic acids and flavonoids: gallic acid, protocatechic acid, gentisic acid, vanillic acid, caffeic acid, chlorogenic acid, syringic acid, pcoumaric acid, ferulic acid, salicylic acid, trans-resveratrol, cis-resveratrol, quercitine (Tables 3, 4). The levels of the different compounds found in wine samples are comparable to those reported in literature. 
244

Among Cypriot wines, 'Maratheftiko' has the higher antioxidant activity (Galanakis et al., 2015); our study has reached the comparable result, which confirms that this method can be used in wine analysis. It should be taken into consideration that the wine is "alive", in a continuous transformation, its composition depends first on the terroir, then on the winemaking techniques or on the storage conditions.

In two of the Romanian wines, 'Frâncusă' R-07-02, 'Fetească regală' R-07-03, but also well-known 'Aligoté' R07-06, quercitine content was under the detection limit, while other phenolics were well represented. Gallic acid, with anti-fungal and anti-viral properties, has the highest concentration in 'Fetească neagră' R-07-16, (6315.54 mg $\mathrm{L}^{-1}$ ) and 'Merlot' C-12-03 (11206.57 $\mathrm{mg} \mathrm{L}^{-1}$ ). Transresveratrol has the highest content in 'Merlot' C-12-03
(1593.06 $\left.\mathrm{mg} \mathrm{L}^{-1}\right)$ and 'Pinot noir' R-07-11 (544.84 $\left.\mathrm{mg} \mathrm{L}^{-1}\right)$. Sources of trans-resveratrol in food include the skin of grapes, blueberries, raspberries, mulberries. Trans-resveratrol provides health benefits, ranging from protection against disease to antiaging properties (Fremont, 2000).

The quantification of the phenolic compounds was performed in order to provide a correlation between DPPH findings and the concentration of the wine samples in phenolic compounds (Table 5). Statistical data underlines the fact that antioxidant capacities by DPPH assay have a strong negative correlation with protocatethic acid $\left(\mathrm{r}_{\mathrm{s}}=\right.$ $-0.78, \mathrm{n}=55, \mathrm{p}<0.001)$, syringic acid $\left(\mathrm{r}_{\mathrm{s}}=-0.73, \mathrm{n}=55\right.$, $\mathrm{p}<0.001)$, trans-resveratrol $\left(r_{s}=-0.70, n=55, p<0.001\right)$ and gallic acid $\left(r_{s}=-0.66, n=55 ; p<0.001\right)$. A moderate negative correlation was observed between the DPPH assay and quercitine $\left(r_{s}=-0.52, n=55, p<0.01\right), n$ being the number of

Table 3. Quantified phenolic compound in Romanian wine sample ( $\mathrm{mg} / \mathrm{L}$ )

\begin{tabular}{|c|c|c|c|c|c|c|c|c|c|c|c|c|c|}
\hline Sample & Gallic acid & $\begin{array}{c}\text { Protocatechuic } \\
\text { acid }\end{array}$ & $\begin{array}{c}\text { Gentisic } \\
\text { acid }\end{array}$ & $\begin{array}{c}\text { Vanillic } \\
\text { acid }\end{array}$ & $\begin{array}{c}\text { Caffeic } \\
\text { acid }\end{array}$ & $\begin{array}{c}\text { Chlorogenic } \\
\text { acid }\end{array}$ & $\begin{array}{c}\text { Syringic } \\
\text { acid }\end{array}$ & $\begin{array}{c}p \text {-coumaric } \\
\text { acid }\end{array}$ & $\begin{array}{c}\text { Ferulic } \\
\text { acid }\end{array}$ & $\begin{array}{c}\text { Salicylic } \\
\text { acid }\end{array}$ & $\begin{array}{c}\text { Trans- } \\
\text { resveratrol }\end{array}$ & $\begin{array}{c}\text { Cis- } \\
\text { resveratrol }\end{array}$ & $\begin{array}{l}\text { Quer- } \\
\text { citine }\end{array}$ \\
\hline R-07-01 & 45.83 & 10.89 & 37.12 & 31.59 & 2094.35 & 0.79 & 3.01 & 215.96 & 663.97 & 320.22 & 4.4 & 1.24 & 10.28 \\
\hline R- $-07-02$ & 22.49 & 12.57 & 4121.34 & 31.96 & 15.15 & 1.39 & 12.78 & 2.19 & 30.95 & 14.28 & 62.09 & 1.16 & \\
\hline R- $-07-03$ & 36.92 & 3.72 & 6492.35 & 11.75 & 14.33 & 2.98 & 7.66 & 3.57 & 96.25 & 23.41 & 7.83 & 1.46 & \\
\hline R- $-07-04$ & 47.1 & 4.97 & 44.62 & 9.64 & 613.48 & 3.06 & 1.28 & 107.71 & 180.58 & 94.04 & 4.88 & 2.06 & 6.11 \\
\hline R- $-07-05$ & 21.58 & 7.47 & 19849.9 & 19.68 & 42.75 & 0.86 & 1.73 & 7.94 & 118.18 & 64 & 8.21 & 1.19 & 1.85 \\
\hline R- $-07-06$ & 14.55 & 5.54 & 5390.19 & 12.27 & 5.27 & 43.46 & 2.35 & 2.43 & 31.03 & 4.88 & 61.46 & 1.4 & \\
\hline R- $-07-07$ & 19.33 & 5.53 & 52.98 & 12.49 & 466.42 & 19.46 & 1.12 & 54.38 & 145.64 & 81.88 & 4.76 & 1.24 & 2.48 \\
\hline R- $-07-08$ & 44.18 & 14.27 & 59.62 & 22.69 & 676.75 & 4.40 & 4.44 & 91.64 & 31.3 & 161.34 & 79.68 & 0.93 & 5.78 \\
\hline R- $-07-09$ & 9 & 5.51 & 15977.09 & 15.08 & 69.03 & 2.84 & 2.12 & 16.68 & 70.55 & 13.45 & 6.79 & 1.03 & 1.31 \\
\hline R- $-07-10$ & 253.04 & 50.3 & 152.91 & 432.21 & 2977.93 & 4.29 & 25.38 & 418.9 & 370.43 & 11.18 & 129.47 & 0.53 & 2.7 \\
\hline R- $-07-11$ & 1003.59 & 52.36 & 7775.16 & 935.05 & 1199.48 & 0.89 & 122.78 & 236.82 & 321.56 & 111.77 & 544.84 & 0.74 & 6.89 \\
\hline R- $-07-12$ & 1041.35 & 30.62 & 6263.23 & 340.99 & 1037.54 & 3.69 & 61.97 & 258.71 & 162.81 & 108.86 & 331.45 & 3.35 & 17.4 \\
\hline R- $-07-13$ & 40.99 & 9.29 & 52.15 & 98.32 & 852.87 & 1.61 & 23.58 & 108.67 & 91.34 & 88.04 & 8.5 & 0.58 & 6.05 \\
\hline R-07-14 & 913.32 & 17.63 & 7148.38 & 137.39 & 387.78 & 4.01 & 41.81 & 145.25 & 152.04 & 56.85 & 169.62 & 1.14 & 9.95 \\
\hline R- $-07-15$ & 272.63 & 33.28 & 16346.53 & 36.65 & 115.87 & 1.5 & 1.63 & 21.71 & 46.03 & 9.13 & 12.45 & 0.31 & 1.18 \\
\hline R- $-07-16$ & 6315.54 & 79.41 & 13686.39 & 594.48 & 562.87 & 28.73 & 211.1 & 201.71 & 45.68 & 60.53 & 471.71 & 0.96 & 3.54 \\
\hline R- $-07-17$ & 439.74 & 28.38 & 5070.44 & 48.21 & 491.33 & 4.83 & 6.24 & 100.98 & 248.94 & 18.63 & 10.28 & 2.89 & 7.33 \\
\hline R- $-07-18$ & 173.38 & 22.62 & 5740.75 & 59.12 & 66.36 & 2.88 & 3.93 & 24.91 & 249.85 & 108.05 & 7.6 & 0.64 & 1.62 \\
\hline R-02-01 & 675.59 & 59.13 & 11506.64 & 40.86 & 264.33 & 5.03 & 5.32 & 68.04 & 129.03 & 4.16 & 11.73 & 0.74 & 9.57 \\
\hline R-02-02 & 405.5 & 33 & 7367.29 & 42.55 & 148.32 & 5.19 & 1.77 & 30.28 & 178.18 & 65.38 & 5.1 & 0.61 & 1.64 \\
\hline R- $-02-03$ & 29.34 & 9.69 & 7445.12 & 39.71 & 53.43 & 13.59 & 2.22 & 13.8 & 194.94 & 108.78 & 14.7 & 0.69 & 1.64 \\
\hline R-02-04 & 235.14 & 8.78 & 2513.45 & 31.46 & 365.16 & 1.05 & 3.51 & 83.27 & 150.3 & 10.18 & 7.76 & 0.63 & 5.71 \\
\hline R- $-02-05$ & 261.54 & 93.13 & 2149.36 & 66.73 & 1613.94 & 22.37 & 5.56 & 389.39 & 171.05 & 17.29 & 66.29 & 1.14 & 24.57 \\
\hline R-02-06 & 422.72 & 19.24 & 14.07 & 34.4 & 1461.89 & 4.64 & 5.16 & 122.69 & 261.11 & 147.12 & 7.64 & 0.7 & 5.26 \\
\hline R- $-02-07$ & 270.7 & 15.56 & 154.32 & 49.57 & 106.98 & 5 & 2.11 & 39.91 & 214.24 & 47.7 & 6.31 & 0.51 & 1.23 \\
\hline R- $-02-08$ & 313.15 & 11.87 & 5047.24 & 29.41 & 387 & 5.73 & 1.87 & 63.13 & 154.72 & 18.48 & 11.41 & 1.16 & 4.26 \\
\hline R-02-09 & 44.59 & 35.04 & 95.84 & 177.21 & 2579.3 & 11.31 & 16.86 & 128.87 & 12.18 & 3.87 & 8.2 & 0.82 & 1.79 \\
\hline R- $-02-10$ & 571.22 & 46.85 & 9815.54 & 116.69 & 194.97 & 8.69 & 29.52 & 84.54 & 90.64 & 21.1 & 38.91 & 0.76 & 6.99 \\
\hline R-02-11 & 2443.12 & 110.85 & 6912.93 & 587.76 & 965.67 & 8.24 & 123.1 & 103.59 & 49.01 & 18.6 & 463.94 & 0.84 & 5.83 \\
\hline R- $-02-12$ & 487.33 & 37.89 & 177.19 & 274.28 & 1093.18 & 6.07 & 46.76 & 225.31 & 37.92 & 3.86 & 47.12 & 0.38 & 1.09 \\
\hline
\end{tabular}

Table 4. Quantified phenolic compounds in Cypriot wine sample ( $\mathrm{mg} / \mathrm{L}$ )

\begin{tabular}{|c|c|c|c|c|c|c|c|c|c|c|c|c|c|}
\hline Sample & Gallic acid & $\begin{array}{l}\text { Protocatechuic } \\
\text { acid }\end{array}$ & $\begin{array}{c}\text { Gentisic } \\
\text { acid }\end{array}$ & Vanillic acid & $\begin{array}{l}\text { Caffeic } \\
\text { acid }\end{array}$ & $\begin{array}{l}\text { Chlorogenic } \\
\text { acid }\end{array}$ & Syringic acid & $\begin{array}{c}P \text {-coumaric } \\
\text { acid }\end{array}$ & Ferulic acid & $\begin{array}{c}\text { Salicylic } \\
\text { acid }\end{array}$ & $\begin{array}{c}\text { Trans- } \\
\text { resveratrol }\end{array}$ & $\begin{array}{c}\text { Tis- } \\
\text { resveratrol }\end{array}$ & $\begin{array}{l}\text { Quer- } \\
\text { citine }\end{array}$ \\
\hline C-13-01 & 30.22 & 18.05 & 1517.74 & 95.31 & 1.97 & 1.92 & 3.02 & 1.59 & 87.95 & 57.73 & 7.50 & 0.34 & 0.75 \\
\hline C-13-02 & 396.27 & 14.25 & 7452.68 & 42.63 & 38.77 & 1.24 & 4.06 & 8.04 & 55.73 & 6.92 & 5.95 & 0.25 & 1.48 \\
\hline C-13-03 & 151.12 & 11.94 & 15009.12 & 71.26 & 29.71 & 1.29 & 1.92 & 3.58 & 42.35 & 7.63 & 19.94 & 0.35 & 1.48 \\
\hline C-13-04 & 388.66 & 12.14 & 12238.09 & 19.43 & 47.63 & 1.76 & 5.56 & 0.48 & 125.46 & 4.77 & 11.00 & 0.22 & 1.01 \\
\hline C-13-05 & 352.03 & 46.29 & 7115.57 & 97.13 & 7.97 & 1.17 & 8.14 & 0.80 & 79.68 & 13.04 & 5.55 & 0.27 & 2.66 \\
\hline C-13-06 & 652.94 & 30.78 & 12264.82 & 28.57 & 20.43 & 0.72 & 5.71 & 3.37 & 48.96 & 6.10 & 38.43 & 0.47 & 2.66 \\
\hline C-13-07 & 484.89 & 11.61 & 7670.02 & 105.73 & 39.12 & 0.72 & 5.53 & 4.04 & 78.28 & 3.75 & 6.92 & 0.21 & 2.23 \\
\hline C-13-08 & 2246.79 & 90.20 & 15526.65 & 548.58 & 3.00 & 0.97 & 50.33 & 5.21 & 11.26 & 304.77 & 814.31 & 24.71 & 320.29 \\
\hline C-13-09 & 1369.01 & 45.49 & 28.42 & 43.67 & 22.11 & 0.89 & 2.84 & 13.28 & 180.63 & 22.52 & 114.44 & 0.78 & 1.03 \\
\hline C-13-10 & 179.81 & 23.98 & 24354.68 & 140.23 & 18.10 & 3.71 & 42.12 & 0.90 & 10.08 & 5.30 & 234.72 & 0.70 & 1.11 \\
\hline C-13-11 & 4805.33 & 62.27 & 11896.78 & 514.50 & 40.39 & 5.78 & 16.75 & 2.44 & 16.51 & 12.46 & 177.86 & 5.47 & 151.59 \\
\hline C-13-12 & 2720.72 & 52.68 & 32042.01 & 353.38 & 77.33 & 87.70 & 30.23 & 3.91 & 9.22 & 279.94 & 1162.87 & 23.90 & 149.54 \\
\hline C-13-13 & 602.27 & 26.65 & 13049.35 & 42.40 & 63.29 & 1.43 & 2.47 & 15.12 & 8.53 & 28.73 & 69.61 & 0.42 & 4.36 \\
\hline C-13-14 & 286.54 & 12.82 & 13704.23 & 7.14 & 37.13 & 3.60 & 2.16 & 2.00 & 47.48 & 5.75 & 10.43 & 0.17 & 4.36 \\
\hline C-13-15 & 208.79 & 34.98 & 17359.95 & 703.58 & 4.69 & 108.83 & 102.83 & 1.90 & 28.61 & 118.73 & 541.50 & 1.59 & 142.50 \\
\hline C-12-01 & 4.81 & 13.72 & 7627.52 & 36.69 & 392.62 & 5.12 & 43.60 & 1.56 & 11.06 & 4.30 & 12 & 0.31 & 4.05 \\
\hline C-12-02 & 960.59 & 42.83 & 7436.28 & 58.17 & 14.38 & 2.30 & 4.09 & 8.04 & 22.82 & 11.05 & 15.41 & 0.65 & 1.78 \\
\hline C-12-03 & 11206.57 & 137.28 & 63.62 & 1122.88 & 80.55 & 48.50 & 116.38 & 5.25 & 27.52 & 291.49 & 1593.06 & 4.73 & 69.38 \\
\hline C-12-04 & 1870.63 & 30.22 & 16.94 & 48.74 & 12.12 & 0.82 & 4.60 & 0.78 & 124.62 & 3.53 & 7.97 & 0.12 & 0.75 \\
\hline C-12-05 & 1493.20 & 59.18 & 8685.72 & 24.30 & 119.81 & 1.10 & 11.88 & 1.15 & 4.91 & 5.07 & 127.02 & 0.51 & 1.38 \\
\hline C-12-06 & 999.01 & 33.04 & 3224.42 & 185.94 & 57.41 & 2.83 & 6.20 & 0.82 & 61.88 & 6.05 & 21.22 & 0.42 & 4.16 \\
\hline C-12-07 & 388.66 & 12.14 & 12238.09 & 19.43 & 47.63 & 1.76 & 5.56 & 0.48 & 125.46 & 4.77 & 11 & 0.22 & 1.01 \\
\hline C-12-08 & 7324.84 & 94.62 & 7877.22 & 988.15 & 117.93 & 3.92 & 94.28 & 3.50 & 4.65 & 116.25 & 549.16 & 5.08 & 150.79 \\
\hline C-12-09 & 4795.94 & 92.40 & 30530.61 & 785.10 & 278.56 & 124.90 & 54.44 & 8.71 & 26.72 & 20.93 & 1320.95 & 7.73 & 15.11 \\
\hline C- $-12-10$ & 8886.82 & 136.28 & 27743.89 & 1326.95 & 245.20 & 162.12 & 100.49 & 8.41 & 18.80 & 752.49 & 900.89 & 56.44 & 148.35 \\
\hline
\end{tabular}


Table 5. Spearmans-Rho coefficient of correlation between antioxidant capacities measured by DPPH assay (EC50) and phenolic compounds of the studied wine

\begin{tabular}{ccc}
\hline Parameter & Spearmans-Rho coefficient & p-value \\
\hline EC50 & 1 & - \\
gallic acid & -0.669 & $<0.001$ \\
protocatechic acid & -0.781 & $<0.001$ \\
\hline gentisic acid & -0.349 & $>0.01$ \\
vanillic acid & -0.212 & $<0.05$ \\
caffeic acid & -0.128 & $>0.5$ \\
chlorogenic acid & -0.302 & $<0.05$ \\
syringic acid & -0.731 & $<0.001$ \\
p-coumaric acid & -0.053 & $<0.05$ \\
\hline ferulic acid & 0.385 & $<0.05$ \\
salicylic acid & -0.211 & $<0.05$ \\
trans-resveratrol & -0.707 & $<0.001$ \\
cis-resveratrol & -0.235 & $<0.05$ \\
quercitine & -0.529 & $<0.01$ \\
\hline
\end{tabular}

samples. These findings suggested that phenolic acids are the most important contributor to antioxidant capacity in these wines.

The highest antioxidant activity for Cypriot white wines was determined at 'Spouriko' wine from 2013, while for Romanian wines the highest value was found in a 'Tămâioasă românească' and for red wines at 'Maratheftiko' wine from 2012 and in 'Fetească neagră' wine from 2014, which demonstrated that the antioxidant activity varies with vintage, grape variety and region (Tables 1 and 2).

\section{Conclusions}

Red wines showed higher antioxidant activity than white or rosé wines. The Spearmans-Rho statistical analysis revealed that the antioxidant capacities determined by $\mathrm{DPPH}$ assay have a strong negative correlation with protocatehic acid, syringic acid, trans-resveratrol and gallic acid and the results presented in this paper can be considered recommendations for consumers who are looking for certain benefits in choosing a wine.

\section{Acknowledgements}

The authors would like to thank the Romanian Executive Agency for Higher Education, Research, Development and Innovation Funding (Romania-Cyprus PN-II-RO-CY-2013-1 project, nr. 764/2014 ROCY WINES "Scientific factors related to consumers' health as new tools for Confirmation of Authenticity of Cypriot/Romanian Wines") and Oenological Research Center of Romanian Academy, Iași Branch, and Ministry of Health State General Laboratory for technical support.

\section{References}

Apak R, Guclu K, Ozyurek M, Karademir SE (2004). Novel total antioxidant capacity index for dietary polyphenols and vitamins $\mathrm{C}$ and $\mathrm{E}$, using their cupric ion reducing capability in the presence of neocuproine: CUPRAC method. Journal of Agricultural and Food Chemistry 52:7970-7981.
Arnous A, Makris DP, Kefalas P (2002). Correlation of pigment and flavanol content with antioxidant properties in selected aged regional wines from Greece. Journal of Food Composition and Analysis 15:655665.

Bell JRC, Donovan JL, Wong R, Waterhouse AL, German JB, Walzen JR (2000). Catechin in human plasma after ingestion of a single serving of reconstituted red wine. American Journal of Clinical Nutrition 71:103108.

Benzie IFF, Strain JJ (1996). The ferric reducing ability of plasma (FRAP) as a measure of "antioxidant power": the FRAP assay. Analytical Biochemistry 239:70-76.

Bondet V, Brand-Williams W, Berset C (1997). Kinetics and mechanisms of antioxidant activity using the DPPH free radical method. LWTFood Science and Technology 30:609-615.

Brand-Williams W, Cuvelier ME, Berset C (1995). Use of a free radical method to evaluate antioxidant activity. Lebensmittel-Wissenschaft \& Technologie 28:25-30.

Bunea CI, Pop N, Babeş AC, Matea C, Dulf FV, Bunea A (2012). Carotenoids, total polyphenols and antioxidant activity of grapes (Vitis vinifera) cultivated in organic and conventional systems. Chemistry Central Journal 6:66.

Cao G, Prior RL (2000). Red wine in moderation: potential health benefits independent of alcohol. Nutrition in Clinical Care 3(2):76-82

Cao GH, Prior R (1999). Measurement of oxygen radical absorbance capacity in biological samples. Methods in Enzymology 299:50-62.

Castellari M, Sartini E, Fabiani A, Arfelli G., Amati A (2002). Analysis of wine phenolics by high-performance liquid chromatography using a monolithic type column. Journal of Chromatography A 973(1-2):221227.

Chang CY, Lee TH, Sheu WHH (2012). Anti-atherogenic effects of resveratrol via liver $\mathrm{X}$ receptor alpha-dependent upregulation of ATPbinding cassette transporters A1 and G1 in macrophages. Journal of Functional Foods 4:727-735.

Cotea DV (1985). Tratat de Oenologie - Vinificaţia şi biochimia vinului [Oenology treatise - Winemaking and biochemistry of wine]. Vol I, Ed Ceres, București.

Cotea DV, Zanoaga VC, Cotea VV (2010). Tratat de Oenochimie [Oenochemistry Treatise]. Editura Academiei Romane.

Cotea VV, Luchian C, Bilba N, Niculaua M, (2012). Mesoporous silica SBA-15, a new adsorbent for bioactive polyphenols from red wine. Analytica Chimica Acta 732:180-185.

Da Porto C, Calligaris S, Celloti E, Nicoli M (2000). Antiradical properties of commercial Cognacs assessed by the DPPH test. Journal of Agricultural Food Chemistry 48:4241-4245.

Fenercioglu AK, Saler T, GencE, Sabuncu H, Altuntas Y (2010). The effects of polyphenol-containing antioxidants on oxidative stress and lipid peroxidation in Type 2 diabetes mellitus without complications. Journal of Endocrinological Investigation 33:118-124.

Floegela A, Kim DO, Chung SJ, KooSI, Chun OK (2011). Comparison of $\mathrm{ABTS} / \mathrm{DPPH}$ assays to measure antioxidant capacity in popular antioxidant-rich US foods. Journal of Food Composition and Analysis 24(7):1043-1048. 
246

Fremont L (2000). Biological effects of resveratrol. Life Sciences 66(8):663673).

Galanakis CM, Kotanidis A, Dianellou M, Gekas V (2015). Phenolic content and antioxidant capacity of Cypriot wines. Czech Journal of Food Sciences 33(2):126-136.

Harris CS, Beaulieu LP, Fraser MH, McIntyre KL, Owen PL, Martineau LC, ... Arnason JT (2011). Inhibition of advanced gycation end product formation by medicinal plant extracts correlates with phenolic metabolites and antioxidant activity. Planta Medica 77(2):196-204.

Hua L, Xiaoyu W, Yong L, Peihong L, Hua W (2009). Polyphenolic compounds and antioxidant properties of selected China wines. Food Chemistry 112:454460.

Katalinić V, Milos M, Modun D, Musić I, Boban M (2004). Antioxidant effectiveness of selected wines in comparison with $(+)$-catechin. Food Chemistry 86:593-600.

Li ASW, Cummings KB, Roethling HP, Buettner GR, Chignell CF (1988). A spin-trapping database implemented on the IBM PC/AT. Journal of Magnetic Resonance 79:140-142.

Llaudy MC, Canals R, Canals JM, Rozés N (2004). New method for evaluating astringency in red wine. Journal of Agricultural and Food Chemistry 52:742-746.

Lopez-Velez M, Martinez-Martinez F, Del Valle-Ribes C (2003). The Study of Phenolic Compounds as Natural Antioxidants in Wine. Critical Reviews in Food Science and Nutrition 43:233-244.

Majo DD, Guardia ML, Giammanco S, Neve LL, Giammanco M (2008). The antioxidant capacity of red wine in relationship with its polyphenolic constituents. Food Chemistry 111:45-49.

Mitić MN, Obradović MV, Grahovac ZB, Pavlović AN (2010). Antioxidant capacities and phenolic levels of different varieties of Serbian whitewines. Molecules 15(3):2016-2027.

Naguib YMA (2000). A fluorometric method for measurement of oxygen radical-scavenging activity of watersoluble antioxidants. Analytical Biochemistry 284:93-98.

Paixao N, Perestrelo R, Marques JC, Camara JS (2007). Relationship between antioxidant capacity and total phenolic content of red, rose and white wines. Food Chemistry 10:204214.
Pulido R, Bravo L, Saura-Calixto F (2000). Antioxidant activity of dietary polyphenols as determined by a modified ferric reducing/antioxidant power assay. Journal of Agricultural and Food Chemistry 48:33963402.

Renaud S, De Lorgeril M (1992). Wine, alcohol, platelets, and the French paradox for coronary heart disease. The Lancet 339(880):1523-1526.

Rotaru L, Jităreanu CD, Cotea VV, Slabu C, Marta AE, Mustea M, Colibaba LC (2013). Adaptarea tehnologiilor viticole în contextul modificărilor climatice [Adapting viticultural technologies to climatic change]. Editura "Ion Ionescu de la Brad", Iaşi.

Saint-Cricq de Gaulejac N, Provost C, Vivas N (1999). Comparative study of polyphenol scavenging activities assessed by different methods. Journal of Agricultural Food Chemistry 47:425-431.

Sánchez-Moreno C, Larrauri JA, Saura-Calixto F (1998). A procedure to measure the antiradical efficiency of polyphenols. Journal of the Science of Food and Agriculture 76:270-276.

Scalbert A, Manach C, Morand C, Rémésy C, Jiménez L (2005). Dietary polyphenols and the prevention of diseases. Critical Reviews in Food Science and Nutrition 45:287-306.

Sun B, Spranger I, Yang J, Leandro C, Guo L, Canano S, Zhao Y, Wu C (2009). Red wine phenolic complexes and their in vitro antioxidant activity.Journal of Agricultural Food Chemistry 57:8623-8627.

Tsai PJ, Huang HP, Huang TC (2004). Relationship between anthocyanin patterns and antioxidant capacity in mulberry wine during storage. Journal of Food Quality 27:497-505.

Xia EQ, Deng GF, Guo YJ, Li HB (2010). Biological activities of polyphenols from grapes. International Journal of Molecular Sciences 11:622-646.

Xu Y, Simon JE, Ferruzzi MG, Ho L, Pasinetti GM, Wu Q (2012). Quantification of anthocyanidins in the grapes and grape juice products with acid assisted hydrolysis using LC/MS. Journal of Functional Foods 4:710-717. 